\title{
O CULTIVO DE HORTA EDUCATIVA NA AMBIENCIA ESCOLAR: UM ESTUDO DE CASO EM UMA REDE MUNICIPAL DE EDUCAÇÃO
}

\author{
Nilsen Aparecida Vieira Marcondes ${ }^{1}$ \\ Edna Maria Querido de Oliveira Chamon ${ }^{2}$ \\ Célia Gomez Sardinha da Silva ${ }^{3}$ \\ Pétala Gonçalves Lacerda ${ }^{4}$
}

Resumo: Considera-se relevante a reflexão sobre o tema horta educativa na ambiência escolar porque se constata a necessidade de resgatar as formas de alimentação saudável que se perderam ao longo do tempo com a introdução de alimentos industrializados, e também sensibilizar as crianças, adolescentes e suas respectivas famílias a cultivarem o hábito da agricultura doméstica. Diante disso, objetiva-se com este estudo básico, qualitativo e descritivo, caracterizado como estudo de caso, refletir sobre o tema no âmbito de algumas escolas municipais em uma cidade do Vale do Paraíba Paulista. Conclui-se, portanto que discutir o tema "O cultivo de horta educativa na ambiência escolar", ensinando por meio de conteúdos pedagógicos e com a prática de uma horta real dentro da escola, além de, contribuir para incentivar os cuidados com o meio ambiente e com a alimentação saudável, possibilita também o desenvolvimento humano, bem como a formação de uma consciência cidadã.

Palavras-chave: Horta Educativa; Ambiência Escolar; Desenvolvimento Humano; Consciência Cidadã.

\footnotetext{
${ }_{1}^{1}$ Programa de Pós-graduação em Educação e Desenvolvimento Humano (PPGEDH)/Universidade de Taubaté (UNITAU), Brasil. E-mail: E-mail: nilsenmarcondes@gmail.com

2 Programa de Pós-graduação em Educação e Desenvolvimento Humano (PPGEDH)/Universidade de Taubaté (UNITAU), Brasil. E-mail: edna.chamon@gmail.com

${ }_{3}^{3}$ Programa de Pós-graduação em Educação e Desenvolvimento Humano (PPGEDH)/Universidade de Taubaté (UNITAU), Brasil. E-mail: cgsds@uol.com.br

${ }^{4}$ Programa de Pós-graduação em Educação e Desenvolvimento Humano (PPGEDH)/Universidade de Taubaté (UNITAU), Brasil. E-mail: petala.lacerda@gmail.com
} 